\title{
The first 500 sacral anterior root stimulators: implant failures and their repair
}

\author{
G S Brindley \\ Spinal Injuries Unit, Royal National Orthopaedic Hospital, Stanmore, HA7 4LP \\ The failures that have occurred in the first 500 sacral anterior root stimulator implants are \\ analysed, and ways of making such failures rarer are discussed.
}

Keywords: sacral anterior root stimulator; spinal cord injury; implant failures

\section{Introduction}

Like other surgically implanted devices, a sacral anterior root stimulator may not survive as long as the patient does. If it fails, it needs to be mended or replaced. This paper reports the failures that have occurred in sacral anterior root stimulator implants during over 1900 patient-years of follow up of the first 500 patients to have them.

When a sacral anterior root stimulator implant that is in use ceases to function, the patient nearly always reports the failure very soon, and asks for something to be done about it. While the implant is not functioning, he or she either does intermittent self catheterisation or has an indwelling catheter inserted. This applies equally whether the failure is in the implant, its external equipment, or the patient's spinal roots or bladder. The inconvenience to the patient of having an unrepaired failure, external or internal, and the simplicity and success of the repair procedures for both, are reasons why it is likely that nearly all failures are reported quickly by the patient to the person who will arrange that the fault will be diagnosed and repaired. Direct evidence that patients usually report failures early comes from routine urological follow ups: I know of no instance, in my clinic or any other, where a patient has come for routine follow up with a previously unreported major implant failure.

\section{External equipment failures}

With every sacral anterior root stimulator the makers supply for the patient's use a test device consisting of a small square coil with its ends connected to a lightemitting diode (LED). When the test device is held over a transmitter that is functioning, the LED flashes. Thus if the stimulator fails, the patient can find out immediately whether the failure is in the external equipment. If it is, Finetech Medical Ltd (or in Germany Ad.Krauth GMBH) will quickly lend temporary external equipment and then repair the patient's own. External equipment failures have not been systematically recorded. It seems that they are commoner than implant failures, but not very much commoner if ageing of the rechargeable batteries and fatigue fractures near the ends of the external cables (two common faults that can be remedied cheaply) is excluded. The present paper is concerned only with internal failures.

\section{Sources of information}

Personal observation is the main source. When an internal failure is reported to the patient's spinal injuries unit, it has been usual, up to August 1994, for the unit to consult me for help with exact diagnosis and treatment. In the future, with growing experience elsewhere, my help will less often be needed. In the 15 years between August 1979 and August 1994, I have taken part in 67 implant repairs excluding implantations of extradural stimulators to replace failed intrathecal ones. I know of nine repairs done or attempted without my help. Six of these nine were done with the recommended equipment, and three with improvised or unorthodox equipment.

Finetech Medical Ltd have sent me a full record of repair equipment sold. From this I know that no repairs using the proper equipment have been done without my knowledge. It is possible that one or two have been done with improvised equipment without my hearing of them.

The questionnaire about the first 500 patients which was sent to all centres ${ }^{1}$ included a question on failures and their repair. Only one failure first came to my notice from an answer to the questionnaire. All the rest had already been reported to me soon after they occurred, usually with a request for my advice or help.

A detailed account of the methods for diagnosing and repairing implant failures is given in reference 2 . The brief outline that follows is needed to explain the observations in the present paper, which were made during repair operations, or on specimens obtained as a result of them. 


\section{Diagnosing the site of a failure}

Sometimes a failure is intermittent, and the implant can be altered from functioning to nonfunctioning or from nonfunctioning to functioning by pressing through the skin on a specific part of it. This gives an excellent indication of the site of failure.

$\mathrm{X}$-ray pictures occasionally show a break in a cable, but most cable failures, and nearly all connector and receiver failures, are radiologically undetectable.

Application of a grid dip meter over a receiver may show lack of resonance at the appropriate frequency, and thus prove that the fault is in the receiver; but most receiver failures leave the radio-frequency resonance unchanged.

\section{Repair procedures}

(a) If a cable failure can be seen in $X$-ray pictures or located by the effects of palpation over the point of failure, and is not very near the receiver block or the place where the cables enter the subarachnoid space A skin incision is made over the site of the failure, roughly parallel to the direction of the cables. The cables are usually exposed with cutting diathermy, which will not damage them. It carries no risk of thermal damage to the trapped spinal roots provided that the diathermy electrode does not directly touch a wire of the cable. Such direct contact at the site of the cable can occur only if all three wires and the insulation are broken right through, or if any of the wires is so bent at the point of failure that it protrudes through the insulation. These kinds of failure show very clearly in $\mathrm{X}$-ray pictures. Therefore if no break in the cable is visible in X-ray pictures, all exposure of cables is done by cutting diathermy. If a break is visible, the broken region is exposed by knife, and then further dissection of cables done by diathermy.

When the faulty region and enough cable on each side of it has been exposed, the faulty region is cut across, and the wires of both stumps exposed by burning.

When exposed, each wire of the lower cable stump in turn is stimulated. If responses are obtained and the threshold is nearly the same for each wire, this establishes that there is no fault distal to the one that was identified preoperatively. All that is needed is to mend the break with a cable-connecting block. If no responses are found, or if the thresholds are very different between wires, a more distal fault must be sought and repaired.

(b) If the failure is known to be in the receiver block or its plug-and-socket connectors, or in cables close to these connectors.

Failures of these kinds can be diagnosed if the channel can be caused to change between functioning and not functioning by digital pressure over the connectors or over the cables near the connectors. Less often, they may be diagnosed from X-ray pictures or by grid dip meter.
The original incision over the receiver block is reopened and the block delivered from its pouch. All three cables are cut close to the connector-jackets if the fault was in the receiver block, or just distal to the fault if the fault was in a cable. The cable ends are burned. Each wire of the lower cable stump is stimulated in turn. If responses are obtained and their thresholds are nearly the same, there is no fault below the one that was identified preoperatively.

All three cables must then be connected to a repair receiver block.

(c) If there is a cable failure within $5 \mathrm{~cm}$ of where the cables enter the subarachnoid space

Such failures are difficult to mend, and the repairs have not proved to be durable. It is better to implant extradural electrodes and connect them to a new receiver block, removing the old receiver block with some or all of its cables. Intrathecal parts of the implant should never be removed unless infected.

\section{(d) If the site of failure is unknown}

One must then proceed according to either (a) or (b). If the wrong choice is made, it wastes time, but does little other harm. The choice should depend on the statistics of kinds of failure. Up to about 1988, the statistics indicated that (b) should be chosen. The experience of recent years, however, favours trying (a) first, because of the rarity, now, of receiver and connector failures.

In a repair procedure, the faulty part of the implant is usually removed and examined. I have done the majority of these examinations. Some were done by $\mathrm{Mr}$ P E K Donaldson or Dr D N Rushton.

\section{Observations}

Failures of the implant

Implants have failed in 72 of the first 500 patients. Fifty-six of these stimulators have been repaired, and nine replaced by new stimulators with extradural electrodes. Five needed no repair, since implant-driven micturition remained good, and two were waiting to be repaired when I last heard about them.

Twenty-one of the 53 repaired stimulators later failed a second time. In 12 of the patients the second failure was repaired, and in nine a new extradural stimulator was implanted. Three of the twelve stimulators that had been repaired twice later failed a third time and were again repaired (2) or replaced (1). Two extradural stimulators put in to replace failed intrathecal ones have failed and been repaired. No failure has yet been reported in any of the 23 patients who had extradural stimulators put in as primary procedures. The total number of known failures is thus $72+21+3+2=98$.

Of all 500 implants, 11 are not known ever to have been used. Four hundred and twenty four were in use at the time of the last follow up known to me, and 65 others had a period of use that was terminated by death of the patient (21) or for other reasons (44). The total time of known use of all implants is 1922.9 years. 
Dividing this by 98 , the number of known implant failures, gives 19.6 years as the best present estimate of the mean time to failure.

First failures have occurred as early as 39 days and as late as 9 years after implantation. Two implants (those of patients 5 and 6 of references 3 and 4 ) have survived 14 years of use without ever needing repair. Table I shows how failures (excluding 11 incidents of failure of material implanted to repair earlier failures) were related to time since implantation. Table II (derived from Table I) shows that the number of failures per 100 implant-years is nearly independent of the time since implantation up to 15 years. It is to be expected that when implants are over 25 years old the silicone rubber will become more brittle, and the failure rate will increase.

\section{Kinds of failure of the implant}

There have been 18 known failures of receivers. In nine of these the component that failed was a resistor of a

Table I Relation of implant failures to time since implantation

\begin{tabular}{lcc}
\hline $\begin{array}{l}\text { Years since } \\
\text { implantation } \\
(n)\end{array}$ & $\begin{array}{c}\text { Number of implants } \\
\text { known to have been } \\
\text { in use at } n \text { years } \\
\text { after implantation }\end{array}$ & $\begin{array}{c}\text { Number of implants } \\
\text { known to have failed } \\
\text { between } n-\frac{1}{2} \text { and } \\
n+\frac{1}{2} \text { years }\end{array}$ \\
\hline 0 & 0 & 3 \\
1 & 410 & 13 \\
2 & 355 & 21 \\
3 & 265 & 10 \\
4 & 189 & 10 \\
5 & 151 & 10 \\
6 & 111 & 11 \\
7 & 74 & 2 \\
8 & 52 & 1 \\
9 & 36 & 5 \\
10 & 26 & 0 \\
11 & 13 & 1 \\
12 & 7 & 0 \\
13 & 6 & 0 \\
14 & 3 & 0 \\
15 & 1 & 0 \\
16 & 1 & \\
\hline
\end{tabular}

Table II Implant failures per 100 implant years (data derived from Table I)

\begin{tabular}{lccc}
\hline $\begin{array}{l}\text { Years since } \\
\text { implantation }\end{array}$ & $\begin{array}{c}\text { Number of } \\
\text { failures }\end{array}$ & $\begin{array}{c}\text { Implant-years } \\
\text { at risk }\end{array}$ & $\begin{array}{c}\text { Failures } \\
\text { per 100 } \\
\text { implant-years }\end{array}$ \\
\hline Less than 0.5 & 3 & about 210 & 1.4 \\
$0.5-1.5$ & 13 & 410 & 3.2 \\
$1.5-2.5$ & 21 & 355 & 5.9 \\
$2.5-3.5$ & 10 & 265 & 3.8 \\
$3.5-4.5$ & 10 & 189 & 5.3 \\
$4.5-5.5$ & 10 & 151 & 6.6 \\
$5.5-6.5$ & 11 & 111 & 9.9 \\
Over 6.5 & 9 & 218 & 4.1 \\
\hline
\end{tabular}

kind that ceased to be used in February 1987. No resistor failure has been detected in any receiver made since that month. The nine other receiver failures were of three different kinds. The manufacturer soon made changes in the manufacturing process designed to make such failures less likely. No failure of any kind has been detected in any receiver made after March 1988.

There are 18 patients in whom plug-and-socket connectors are known to have failed. The design of these was improved in May 1987. Of the 18 patients with failures, 15 had unimproved connectors, made before May 1987. In 11 of the 18 patients, a single plug or single socket had failed, out of the nine plugs and nine sockets in a three-channel receiver block. In four patients two plugs or sockets had failed. In three patients, five, seven and 15 of the 18 plugs and sockets had failed. Multiple failures may be due to unusual behaviour by the patient, perhaps pressing the transmitter block very strongly onto the implanted receiver block.

In three patients, $\mathrm{X}$-ray pictures taken to help diagnosis of a failure showed that the receiver block was folded within its subcutaneous pouch. The folding may have long preceded the failure that it ultimately caused. The failure was in each case a fracture of the short length of cable between a receiver and its plug connector.

All other known failures for which a diagnosis was possible ( 42 in number) have been of cables outside the receiver block. The design of the cables has not altered significantly since 1973, when sacral anterior root stimulators were in use only in experimental animals. Cable failures seem to be about as common in recent implants as they were (at the same length of follow up) in older ones. The cables are extremely resistant to flexion and to stretching, ${ }^{5}$ but less resistant to lateral compression. When they are experimentally subjected to repeated lateral compression, and suffer multiple breaks, these breaks always form longitudinal rows in one turn or many consecutive turns of the triple helix. This longitudinal row pattern is found in nearly all breaks examined in cables removed from patients.

In 19 patients, cable failures have been proved by surgical exploration to be near the midline of the back, close enough to the exit from the dura to make repair impossible (14 cases) or difficult (five cases). In one of the 14 where repair was impossible, the grommet designed to prevent leakage of cerebrospinal fluid had fractured, and all three cables had failed in the plane of the fracture. In three of the five cases where repair was possible but difficult, a new failure appeared at the join within 2 years. In the other two the repair is still good after 5 years and 1 year. Five of the 11 patients with failures near the midline of the back described habitual actions, either domestic (3) or in playing basketball (2), which would have compressed the cables between the back of the wheelchair and bony structures.

Nine cable failures were at sites easily accessible by reopening the incision through which the receiver block was implanted. All these failures were less than $3 \mathrm{~cm}$ in a straight line from the receiver block, and were within 
the segment of cable that had been placed in the receiver pouch by the surgeon. The distance from the plug-and-socket joint as measured along the cable varied between 2 and $16 \mathrm{~cm}$. In one of these nine failures, microscopical examination of the specimen showed a clean planar cut through half the cross-section of the rubber and a tear through the other half, and the wire fractures were in the transverse plane of the rubber failure, not in the usual longitudinal row. Almost certainly this cable has suffered a knife injury at the time of implantation, and subsequently failed by repeated flexion at the site of the injury.

The nine cable failures close to the receiver block were all in patients (about 340 in number) who had their receivers in the thoracic site that is usual in the UK, France and most other countries. There were no such failures among the approximately 160 patients whose receivers were in the abdominal site favoured in Germany and the Netherlands.

In one patient all three cables failed where they passed over the left 10 th rib. In all three cables the breaks in the wires formed longitudinal rows. The patient used a wheelchair with armrests, had scoliosis concave to the left, and had the habit of leaning on his left armrest. It is almost certain that the breaks were due to compression of the cables between the armrest and the 10th rib. In all, seven patients with cable failures have described habitual actions which would have been likely to compress cables against a bony structure near where the failure was found, and it is not unlikely that such compression accounts for all or nearly all cable failures.

For seven failures, repair procedure (b) showed no responses to stimulation through the wires of the distal burnt stump, and it was decided to implant a new extradural stimulator without further exploration. The faults must have been cable failures remote from the receiver block.

In all, there have been five known cable failures among 122 tetraplegic patients (4.1\%) and 37 among 264 paraplegic patients $(14.0 \%)$. The higher proportion of failures in paraplegics $\left(\chi^{2}=8.03\right.$ for $\left.1 \mathrm{df}, p<0.01\right)$ is not surprising, since on average they are more active.

No cable failure has yet been detected in an extradural implant. It is theoretically likely that paravertebral cable failures will be less common in extradural implants.

\section{Failures of the spinal roots and bladder}

If an anterior spinal root fails, its somatic and autonomic functions usually (perhaps always) fail together. Failures of roots in the first 2 weeks after implantation are already well known. ${ }^{3,4,6}$ The damage probably occurs during the operation, but is not detected until Wallerian degeneration of the damaged fibres occurs about a week later. Recovery is usual, but takes months.

In eight patients, the function of roots deteriorated more than 2 weeks after implantation. In one man the deterioration was sudden, and due to a fracture- dislocation of lumbar vertebrae, probably secondary to osteomyelitis. In all the rest the deterioration was gradual, during several months. In one it was due to infection of the implant with an organism of low virulence. It was later necessary to remove the whole of this implant. In two it was attributed to a descending syrinx. One patient had multiple sclerosis, and the deterioration may have been due to her disease. In three, aged 37,54 and 64 , root function failed gradually after 7 months, 6 years and 7 years respectively of good implant-driven micturition, without any known reason. The 54 year old man died a year later from hepatitis; the 37 year old man and the 64 year old woman remain well, and are continent on intermittent self catheterisation.

\section{Isolated failure of bladder responses}

Among the patients whom I know well, five have had episodes of failure of implant-driven micturition during which all the skeletal muscle responses to the implant remained unchanged. Four of these patients were users of the implant for defaecation, and they found that for this purpose it continued to work though it had failed for micturition. These episodes followed incidents during which the bladder probably became very overfull. It is likely that the failures were due to overstretching of nerve fibres in the wall of the bladder. The bladder responses recovered after a few weeks in all five patients.

\section{Discussion}

Failures of receivers and of plug-and-socket connectors have been made much rarer by changes in design made in 1987. The solitary failure of a grommet also provoked a change in design which is likely to prevent a similar failure in any implant made after March 1993. Cable failures remain worrying. However, a mean time to failure of 19.6 years is not very bad, especially as it includes receiver and connector failures, which were formerly common but are now rare. It is almost certain that cable failures can be diminished by changes in the procedure of implantation, especially the routing of cables as far as possible from bone.

It is uncertain whether any practicable change in the design of the implant would help. Cables that are less vulnerable to lateral compression than those of the Finetech-Brindley bladder controller can be made, without sacrificing the excellent ability of the present cables to withstand flexion. But cables with these properties (widely used in cardiac pacemakers) are inextensible, and the harm from their inextensibility might outweigh the gain from avoiding lateral compression failures.

\section{References}

1 Brindley GS (1994) The first 500 patients with sacral anterior root stimulator implants: general description. Paraplegia 32: 795-805. 
2 Brindley GS (1994) The Finetech-Brindley Bladder Controller: Notes for Surgeons and Physicians. Finetech, Welwyn Garden City.

3 Brindley GS, Polkey CE, Rushton DN, Cardozo L (1986) Sacral anterior root stimulators for bladder control in paraplegia: the first 50 cases. J Neurol Neurosurg Psychiatry 49: 1104-1114.

4 Brindley GS, Rushton DN (1990) Long-term follow-up of patients with sacral anterior root stimulator implants. Paraplegia
28: $469-475$

5 Donaldson PEK (1983) The Cooper cable: an implantable multiconductor cable for neurological prostheses. Med Biol Eng Comput 21: 371-374.

6 Egon G, Colombel P, Isambert J et al (1993) Evolution of bladder contraction in course of time after implantation of a sacral anterior root stimulator. Neurourol Urodynam 12: 509-512. 